\title{
LES MONDES MÊLÉS DU COMMUNISME : UNE AUTRE APPROCHE TRANSNATIONALE ?
}

\author{
Paul Boulland, Isabelle Gouarné
}

Presses de Sciences Po (P.F.N.S.P.) | « Critique internationale»

2015/1 N 66 | pages 9 à 18

ISSN 1290-7839

ISBN 9782724633962

Article disponible en ligne à l'adresse :

http://www.cairn.info/revue-critique-internationale-2015-1-page-9.htm

\section{Pour citer cet article :}

Paul Boulland, Isabelle Gouarné, « Les mondes mêlés du communisme : une autre approche transnationale? », Critique internationale 2015/1 (N66), p. 9-18. DOI 10.3917/crii.066.0009

Distribution électronique Cairn.info pour Presses de Sciences Po (P.F.N.S.P.).

(C) Presses de Sciences Po (P.F.N.S.P.). Tous droits réservés pour tous pays.

La reproduction ou représentation de cet article, notamment par photocopie, n'est autorisée que dans les limites des conditions générales d'utilisation du site ou, le cas échéant, des conditions générales de la licence souscrite par votre établissement. Toute autre reproduction ou représentation, en tout ou partie, sous quelque forme et de quelque manière que ce soit, est interdite sauf accord préalable et écrit de l'éditeur, en dehors des cas prévus par la législation en vigueur en France. Il est précisé que son stockage dans une base de données est également interdit. 


\section{Les mondes mêlés du communisme : une autre approche transnationale?}

par Paul Boulland et Isabelle Gouarné

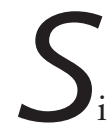

i l'on ne compte plus désormais les ouvrages, numéros de revues, colloques et séminaires consacrés au « tournant global » des sciences humaines et sociales, le débat reste ouvert sur la véritable portée de ce changement et sur les transformations qu'il appelle dans le régime des savoirs : certains considèrent qu'il importe maintenant de définir le nouveau paradigme sous-jacent aux global studies; d'autres, en revanche, se méfiant des effets de mode, soulignent le flou conceptuel et méthodologique de ces nouvelles approches et émettent des doutes sur le potentiel heuristique qu'on leur attribue dans presque tous les champs de recherche1. L'ampleur de la discussion invite aujourd'hui à documenter les mutations d'objets, de méthodes et de questionnements déjà engagées dans différents domaines. C'est dans ce mouvement réflexif que souhaite s'inscrire ce dossier : les articles réunis ici participent tous en effet d'une histoire sociale ou socio-histoire des communismes ${ }^{2}$ qu'ils entendent éclairer à l'aide des outils analytiques des approches «transnationales », développées sous les différents labels d'histoires « globale », « connectée » ou encore « croisée ».

1. Parmi les publications récentes sur ce débat, voir Alain Caillé, Stéphane Dufoix (dir.), Le tournant global des sciences sociales, Paris, La Découverte/Bibliothèque du MAUSS, 2013 ; Geoffrey Pleyers, « Sociologie de la mondialisation. Au-delà des globalistes et des sceptiques », Recherches sociologiques et antbropologiques, 2012, horssérie, p. 105-123 ; Antoine Vauchez, « Le prisme circulatoire. Retour sur un leitmotiv académique », Critique internationale, 59, avril-juin 2013, p. 9-16 ; « AHR Conversation: On Transnational History », The American Historical Review, 111 (5), 2006, p. 1440-1464.

2. Pour une synthèse, voir Michel Dreyfus et al., Le siècle des communismes, Paris, Le Seuil, 2004 (2000). 
L'enjeu ici n'est pas de discuter les apports respectifs de ces approches sensiblement différentes, mais, plus modestement, de considérer leur commune orientation pour en examiner les effets sur un objet de recherche - le communisme - dont la dimension internationale a été d'emblée fortement interrogée, en raison notamment des représentations politiques qui y étaient associées (« l'œil de Moscou », le parti communiste comme « parti de l'étranger »...). Des questions telles que les rapports des différents partis communistes avec « Moscou », l'« influence »de l'Union Soviétique dans le monde et les différents instruments (diplomatiques ou « officieux ») de sa politique étrangère ${ }^{3}$ ou, à l'inverse, les emprunts occidentaux du communisme soviétique ${ }^{4}$ et son « américanisation » ont fait, depuis longtemps déjà, l'objet de travaux importants qui peuvent être aujourd'hui relus, critiqués, repensés ou prolongés dans une perspective transnationale.

Depuis les années 2000, cette orientation a suscité un tel engouement dans les différents secteurs de recherche que recoupe l'histoire sociale des communismes (sociologie historique des partis et organisations communistes, histoire sociale des pays socialistes, relevant donc de diverses aires culturelles) qu'il serait impossible d'en dresser ici le panorama ${ }^{5}$. Elle a été un moyen de repenser la dimension internationale du communisme, en sortant de toute vision diffusionniste, focalisée sur l'État soviétique et ses relais, pour poser le regard sur les circulations d'hommes, d'idées, de savoirs, de pratiques, de modèles et de représentations entre les pays socialistes et, au-delà, avec l'Occident et «les Suds ». De fait, les approches développées sont très diverses : elles peuvent prendre des objets de recherche déjà donnés comme internationalisés (comme c'est le cas, par exemple, de la socio-biographie des «go-between » ou de l'étude institutionnelle des organisations médiatrices); elles peuvent aussi s’ancrer dans des études localisées et centrées sur les pratiques (histoire

3. Voir, par exemple, Annie Kriegel, Georges Haupt, « Les groupes communistes étrangers en Russie et la Révolution mondiale (1917-1919). État des travaux », Revue d'bistoire moderne et contemporaine, 10 (4), 1963 , p. 289-300 ; Annie Kriegel, Stéphane Courtois, Le système communiste mondial, Paris, PUF, 1984 ; A. Kriegel, S. Courtois, Eugen Fried: le grand secret du PCF, Paris, Le Seuil, 1997.

4. Voir, notamment, les nombreuses recherches menées, dès les années 1970-1980, sur l'organisation du travail en URSS et ses rapports au taylorisme : entre autres, Samuel Lieberstein, « Technology, Work, and Sociology in the USSR: The NOT Movement », Technology and Culture, 16 (1), janvier 1975, p. 48-66 ; Robert Linhart, Lénine, les paysans, Taylor, Paris, Le Seuil, 1976 ; Zenovia A. Sochor, « Soviet Taylorism Revisited », Soviet Studies, 33 (2), avril 1981, p. 246-264 ; Mark R. Beissinger, Scientific Management, Socialist Discipline, and Soviet Power, Cambridge, Harvard University Press, 1988.

5. Depuis 2013, le séminaire «Sociétés communistes et post-communistes », organisé par Sophie Cœuré et Taline Ter Minassian (Université Paris 7/INALCO), s'inscrit dans cette perspective et s'intéresse précisément aux « circulations politiques et culturelles entre l'Europe "de l'Ouest" et l'Europe "de l'Est" au $\mathrm{XX}^{\mathrm{e}}$ siècle ». Pour une mise au point récente sur l'état de la recherche, voir Sari Autio-Sarasmo, Katalin Miklossy (eds), Reassessing Cold War Europe, Londres/New York, Routledge, 2011 ; Michael David-Fox, « The Implications of Transnationalism », Kritika: Explorations in Russian and Eurasian History, 12 (4), 2011, p. 885-904 ; Justine Faure, Sandrine Kott (dir.), « Le Bloc de l'Est en question », dossier, Vingtième Siècle. Revue d'bistoire, 109, 2011 ; György Péteri, « Nylon Curtain - Transnational and Transsystemic Tendencies in the Culture Life of State-Socialist Russia and East-Central Europe », Slavonica, 10 (2), novembre 2004, p. $113-123$. 
des techniques de pouvoir, histoire du quotidien des régimes communistes, sociologie du militantisme). Leur point commun est l'attention portée au contenu de ce qui circule, aux transformations que ce contenu subit en se déplaçant, aux appropriations, aux réceptions et aux usages qu'il connaît dans des contextes différents.

Des questions de recherche anciennes ont été ainsi renouvelées, et d'abord celle, longtemps très polémique, de l'« influence » soviétique à l'étranger. Les approches en termes de « circulation d'idées » et de « transferts culturels $»^{6}$ ont, par exemple, permis de rompre avec les problématiques, héritées du paradigme totalitariste, de la «manipulation » et de l'« aveuglement »des intellectuels occidentaux. La réflexion s'est déplacée vers les médiateurs, les institutions relais et les effets des interactions Est-Ouest dans la construction des représentations politiques, des savoirs et des idées ${ }^{7}$. Avec l'ouverture des archives en Russie, par exemple, la diplomatie culturelle mise en œuvre par l'État soviétique à partir des années 1920 a fait l'objet de nouvelles recherches étudiant les multiples appropriations de « la culture soviétique » qu'elle avait rendues possibles en Occident et qui avaient ainsi influé sur les espaces intellectuels européens ou américains ${ }^{8}$.

Une même orientation est aujourd'hui suivie pour analyser les relations des différents partis communistes nationaux avec Moscou sans les réduire à de simples rapports de soumission ou d'inféodation. La formation d'un espace politique communiste relativement homogène résulterait de la mise en circulation d'idées, de savoirs et de pratiques qui, certes, furent mobilisés selon un référentiel commun, mais qui, en se déplaçant, connurent de multiples transformations, furent soumis à

6. Pierre Bourdieu, «Les conditions sociales de la circulation internationale des idées », Actes de la recherche en sciences sociales, 145, 2002, p. 3-8 ; Michel Espagne (dir.), Transferts culturels triangulaires France-Allemagne-Russie, Paris, Éditions de la MSH, 1996 ; Michael Werner, Bénédicte Zimmermann, « Penser l'histoire croisée : entre empirie et réflexivité », Annales. Histoires, Sciences sociales, 1, 2003, p. 7-36; M. Werner, B. Zimmermann (dir.), De la comparaison à l'bistoire croisée, Paris, Le Seuil, 2004.

7. De nombreux travaux ont été consacrés ces dernières années aux circulations culturelles Est-Ouest. Dans le domaine scientifique, voir notamment Susan Gross Solomon (ed.), Doing Medecine Together. Germany and Russia between the Wars, Toronto, University of Toronto Press, 2006 ; Michael David-Fox, Peter Holquist, Alexander Martin (eds), «Circulation of Knowledge and the Human Sciences in Russia », Kritika: Explorations in Russian and Eurasian History, 9, 2008 ; Grigorij M. Bongard-Levin, Roland Lardinois, Aleksej A. Vigasin, Correspondances orientalistes entre Paris et Saint-Pétersbourg (1887-1935), Paris, Mémoires de l'Académie des Inscriptions et Belles-lettres, 2002. Dans le domaine littéraire, voir entre autres Katerina Clark, Moscow, The Fourth Rome: Stalinism, Cosmopolitanism, and the Evolution of Soviet Culture, 1931-1941, Cambridge, Harvard University Press, 2011 ; Ioana Popa, Traduire sous contrainte. Littérature et communisme (1947-1989), Paris, CNRS Éditions, 2010.

8. Sur la diplomatie culturelle soviétique, voir, parmi les publications récentes, S. Cœuré, La grande lueur à l'Est. Les Français et l'Union Soviétique, 1917-1939, Paris, Le Seuil, 1999 ; Sophie Couré, Rachel Mazuy (dir.), Cousu de fil rouge. Voyages des intellectuels français en Union Soviétique, Paris, CNRS Éditions, 2012 ; M. DavidFox, Showcasing the Great Experiment. Cultural Diplomacy and Western Visitors to the Soviet Union, 1921-1941, Oxford, Oxford University Press, 2012 ; Jean-François Fayet, VOKS Le laboratoire helvétique. Histoire de la diplomatie culturelle soviétique durant l'entre-deux-guerres, Chêne-Bourg, Georg Éditeur, 2014 ; Isabelle Gouarné, L'introduction du marxisme en France. Philosoviétisme et sciences humaines, 1920-1939, Rennes, Presses universitaires de Rennes, 2013. 
des (ré)appropriations variées et eurent, en retour, des effets sur « le centre ${ }^{9}$. Cette perspective de recherche a été notamment développée à partir d'une réflexion sur les pratiques biographiques et autobiographiques (« autobiographies communistes d'institution », aveux, récits édifiants...) qui caractérisent le monde communiste $^{10}$. À travers les dispositifs institutionnels qui permirent leur généralisation et les différentes appropriations que connurent ces pratiques en URSS, dans l'Internationale communiste puis dans les différents partis communistes et, après la guerre, en Europe de l'Est, c'est le système communiste « dans ce qu'il a de spécifiquement "nouveau" $\gg^{11}$ qui est interrogé.

Cette question de la spécificité du communisme est aussi retravaillée à l'échelle des «blocs » et de leurs « frontières $»^{12}$. Au lieu d'examiner les luttes et les rivalités entre l'Est et l'Ouest, il s'agit désormais d'étudier comment cette opposition s'est construite et imposée jusqu'à dissimuler les interconnexions, les échanges, les coopérations entre les deux systèmes socio-politiques. L'histoire du communisme au quotidien, avec des objets d'étude centrés sur la consommation, les loisirs ou le travail, montre ainsi que les spécificités socialistes, même celles relatives aux pratiques du pouvoir et de l'autorité, se produisent localement à partir de circulations transnationales ${ }^{13}$.

En dépassant les cadrages nationaux et disciplinaires traditionnels, la perspective transnationale a donc contribué à décloisonner l'étude des régimes, des organisations et des partis communistes, et à les insérer dans des histoires globales (du travail, de l'économie, du pouvoir, de la culture et des sciences...). Désormais, le monde communiste, dans ses différentes composantes, est perçu comme un lieu d'interactions, aussi bien pour la période des années 1920 et celle du Dégel

9. C'est dans cette perspective que travaille notamment Jérémie Tamiatto dans le cadre de sa thèse (en voie d'achèvement) intitulée « Les missionnaires de la révolution en Chine 1920-1927, étude socio-politique des envoyés du Komintern ». Sur la réception du modèle soviétique en Chine, voir aussi Thomas P. Bernstein, Hua-Yu Li (eds), China Learns from the Soviet Union, 1949-Present, Plymouth, Lexington Books, 2010. Pour l'Europe, voir, par exemple, Bert Hoppe, « Iron Revolutionaries and Salon Socialists: Bolsheviks and German Communists in the 1920's and 1930's », Kritika: Explorations in Russian and Eurasian History, 10 (3), 2009, p. 499-526. Sur les acteurs de l'Internationale communiste, voir José Gotovitch, Mikhaïl Narinski (dir.), Komintern : l'histoire et les hommes. Dictionnaire biographique de l'Internationale communiste, Paris, Éditions de l'Atelier, 2001.

10. Claude Pennetier, Bernard Pudal (dir.), Autobiographies, autocritiques, aveux dans le monde communiste, Paris, Belin, 2002 ; C. Pennetier, B. Pudal (dir.), Le sujet communiste. Identités militantes et laboratoires du « moi », Rennes, Presses universitaires de Rennes, 2014.

11. C. Pennetier, B. Pudal, « Avant-propos. Un monde biographique nouveau », dans C. Pennetier, B. Pudal (dir.), Autobiographies, autocritiques, aveux dans le monde communiste, op. cit., p. 9.

12. Sophie Cœuré, Sabine Dullin (dir.), Les frontières du communisme, Paris, La Découverte, 2007.

13. Voir notamment Yves Cohen, Le siècle des chefs. Une bistoire transnationale du commandement et de l'autorité (1890-1940), Paris, Éditions Amsterdam, 2013 ; Anne Gorsuch, All This Is Your World. Soviet Tourism at Home and Abroad after Stalin, Oxford, Oxford University Press, 2011 ; G. Péteri (ed.), « Sites of Convergence. The USSR and Communist Eastern Europe at International Fairs Abroad and at Home », Journal of Contemporary History, 47, janvier 2012 ; Larissa Zakharova, S'habiller à la soviétique. La mode et le Dégel en URSS, Paris, CNRS Éditions, 2011 ; L. Zakharova (dir.), « Le quotidien du communisme », Annales. Histoire, sciences sociales, 2, avriljuin 2013. 
khrouchtchévien ${ }^{14}$, considérées généralement comme des moments d'ouverture, que pour la période stalinienne et celle de la guerre froide, considérées comme des moments de crispation politique et idéologique ${ }^{15}$. Toutes les aires culturelles sont également concernées. Certes, les recherches se sont davantage centrées jusqu’à présent sur les interactions Est-Ouest, mais le monde « tricontinental (l'Afrique, l'Asie, l'Amérique latine) devient aussi un terrain d'exploration privilégiée ${ }^{16}$.

Pour autant, et bien que foisonnante, cette tendance historiographique peut avoir quelque chose de déroutant. C'est en effet avec l'effondrement des régimes communistes que les termes de « globalisation », «mondialisation » ou « circulation » ont conquis les sciences sociales, à la fois pour penser le nouvel ordre planétaire et pour désigner une nouvelle orientation méthodologique refusant le compartimentage national et l'ethnocentrisme occidental. S'il convient sans doute de mieux faire la distinction entre ces deux aspects, il reste que les approches globales se sont développées à partir d'une réflexion sur la disparition des États, l'effacement des frontières nationales et le devenir des sociétés libérales. C'est dire combien le recours à ce type d'approches pour analyser les phénomènes communistes du $\mathrm{XX}^{\mathrm{e}}$ siècle n'allait pas de soi. On le verra à la lecture des articles réunis ici qui témoignent des renouvellements engagés dans l'histoire et la sociologie des communismes, précisément sur des périodes (la seconde guerre mondiale et la guerre froide) et des régions (les interactions au sein du bloc de l'Est et avec l'Ouest) pour lesquelles l'inscription dans une perspective transnationale pouvait initialement paraître contre-intuitive. En ce sens, ce dossier répond au souhait exprimé par Heinz-Gerhard Haupt qui, dénonçant « le préjugé positif de l'échange et de la circulation », invitait à analyser « des milieux, des conjonctures et des

14. Sur les renouvellements historiographiques récents concernant la période du Dégel, voir Eleonory Gilburd, Larissa Zakharova (dir.), «Repenser le Dégel. Versions du socialisme, influences internationales et société soviétique », Cahiers du monde russe. Russie, Empire russe, URSS, États indépendants, 47 (1-2), 2006. Sur la période postérieure, voir Marc Elie, Isabelle Ohayon (dir.), « L'expérience soviétique à son apogée. Culture et société des années Brejnev », Cahiers du monde russe. Russie, Empire russe, URSS, États indépendants, 54 (1-2), 2013.

15. Sur la période stalinienne, voir notamment Y. Cohen, « Les localités circulatoires. L'exemple du haut stalinisme dans les années $1930 »$, Les Cabiers du Centre de recherches historiques, 42, 2008, p. 253-282. Sur les nouvelles interprétations « globales » de l'histoire de la guerre froide, voir, entre autres, S. Autio-Sarasmo, K. Miklossy (eds), Reassessing Cold War Europe, op. cit..

16. Marie-Pierre Rey (dir.), «L'URSS et le Sud», Outre-mers, 94 (354-355), 2007 ; Christine Philliou (ed.), «USSR South. Postcolonial Worlds in the Soviet Imaginary », Comparative Studies of South Asia, Africa and the Middle East, 33 (2), 2013. Sur l'Afrique, voir notamment Constantin Katsakioris, « L'Union soviétique et les intellectuels africains. Internationalisme, panafricanisme et négritude pendant les années de la décolonisation, 1954-1964 », Cahiers du monde russe. Russie, Empire russe, URSS, États indépendants, 47 (1-2), 2006, p. 15-32 ; C. Katsakioris, « Soviet Lessons for Arab Modernization. Soviet Educational Aid Towards Arab Countries after 1956 », Fournal of Modern European History, 1 (8), mars 2010, p. 85-105 ; Françoise Blum, Révolutions africaines. Congo, Sénégal, Madagascar, années 1960-1970, Rennes, Presses universitaires de Rennes, 2014. Voir également le projet ELITAF («Élites africaines formées en URSS et dans les autres pays de l'ancien bloc soviétique. Histoires, biographies, expériences »), coordonné par Michèle Leclerc-Olive, Monique de Saint-Martin et Patrice Yengo. Sur l'Asie, voir, par exemple, les recherches de David C. Engerman : « Learning for the East: Soviet Experts and India in the Era of Competitive Coexistence », Comparative Studies in South Asia, Africa and the Middle East, 33 (2), 2013, p. 227-238 ; D. C. Engerman « The Political Power of Economic Ideas ? Foreign Economic Advisors and Indian Planing in the 1950's and 1960's », dans Andreas Hilger, Corinna R. Unger (eds), India in the World since 1947. National and Transnational Perspectives, Berne, Peter Lang, 2012, p. 120-135. 
contextes qui ont été résistants à la circulation et aux contacts externes $\gg^{17}$. Une telle démarche permet de fait de revenir sur certains présupposés et implicites des approches globales.

\section{Reconsidérer le rôle de l'État dans les processus transnationaux}

La montée du «transnational » dans le champ académique, à la fin des années 1980 et au début des années 1990, concernait, en premier lieu, le domaine des relations internationales qui connut ainsi une nouvelle actualité scientifique. Développée d'abord aux États-Unis, la perspective transnationale désignait une façon d'envisager les relations internationales, en rupture avec l'approche « réaliste », stato-centrée $^{18}$. Dès lors, afin de mettre au jour des formes d'échanges et de flux indépendants des États, on étudia de préférence les migrations internationales, les ONG, les réseaux militants internationaux ou encore les fondations américaines. L'intérêt heuristique de ces nouvelles approches n'est plus contesté aujourd'hui. Leur risque, cela a été maintes fois souligné, fut néanmoins de surestimer la dénationalisation des configurations ainsi étudiées. L'un des principaux axes de réflexion a de ce fait porté sur les modes d'emboîtement des échelles (locale, nationale, internationale). C'est donc d'abord sur cette question que revient ce dossier, dont le parti pris méthodologique transnational conduit paradoxalement à souligner la prégnance des logiques nationales.

Telle est la conclusion à laquelle parvient Michel Christian après s'être interrogé sur l'existence au sein du «bloc de l'Est »d'une dimension transnationale relativement indépendante des enjeux diplomatiques traditionnels. Pour rendre compte de la densité des échanges horizontaux entre les partis communistes de l'Europe socialiste, il observe les jumelages mis en place au niveau local, à partir des années 1950, entre les partis communistes de RDA et de Tchécoslovaquie. Or il apparaît que, s'ils résultaient parfois d'initiatives individuelles et locales, ces partenariats furent très rapidement pris dans des intérêts nationaux et perçus avant tout comme des instruments diplomatiques : du côté est-allemand, pour pouvoir affirmer la légitimité du nouvel État à représenter l'Allemagne ; du côté tchécoslovaque, pour faire reconnaître les accords de Postdam de juillet 1945. D'ailleurs, dès que ces jumelages furent susceptibles de constituer une menace pour la légitimité de l'État socialiste, comme en 1968, au moment du Printemps de Prague, ils furent interrompus.

17. Heinz-Gerhard Haupt, « Une nouvelle sensibilité : la perspective "transnationale". Une note critique », Cabiers faurès, 200(2), 2011, p. 178.

18. Pour une présentation de ce mouvement historiographique, voir S. Dufoix, « Les naissances académiques du global », dans A. Caillé, S. Dufoix (dir.), Le tournant global des sciences sociales, op. cit., p. 27-43 ; Pierre Grosser, «L'histoire mondiale/globale, une jeunesse exubérante mais difficile », Vingtième Siècle. Revue d'bistoire, 110, 2011, p. 3-18. ; Chloé Maurel, « La World/Global History. Questions et débats », Vingtième Siècle. Revue d'bistoire, 104, 2009, p. 153-166 ; Pierre-Yves Saunier, « Transnational », dans Akira Iriye, Pierre-Yves Saunier (eds), The Palgrave Dictionary of Transnational History, Basingstoke, Palgrave Macmillan, 2009, p. 1047-1055. 
Le même constat relatif à la prééminence du national est fait par Alix Heiniger. Son enquête porte sur les membres du Parti communiste allemand (KPD) qui, après avoir émigré en Europe de l'Ouest, rejoignirent les sections françaises, belges et suisses du mouvement Freies Deutschland (Allemagne libre), créé à Moscou en juillet 1943. Leur socialisation politique antérieure au sein du mouvement communiste, via leur adhésion au KPD dans les années 1920 ou 1930, voire, pour certains, leur passage par l'Internationale communiste à Moscou, facilitèrent grandement, durant la seconde guerre mondiale, leur collaboration avec des militants communistes d'autres nationalités : tous partageaient le même discours internationaliste de lutte contre le fascisme et les mêmes savoir-faire militants. L'internationalisme, comme discours et pratiques inventés et diffusés par l'Internationale communiste, semble donc, dans ce contexte de guerre, avoir eu des effets positifs sur les interactions militantes transnationales. Pourtant, en 1943, la création de Freies Deutschland initia un autre militantisme, tourné vers la construction d'une nouvelle Allemagne « libre et démocratique ». Cette réorientation se traduisit concrètement par des pratiques et des discours militants visant la communauté nationale et non plus la communauté politique.

Sans doute n'est-il plus nécessaire de revenir sur le présupposé d'une exclusion réciproque du national et du transnational, tant il a été discuté et empiriquement critiqué ces dernières années. De fait, les différents niveaux d'analyse ne sont pas exclusifs les uns des autres et étudier les interactions entre processus nationaux et transnationaux, hier comme aujourd'hui, est devenu l'un des enjeux les plus stimulants des recherches actuelles sur la «globalisation $»^{19}$. Au terme de leurs analyses, les auteurs de ce dossier ne proposent donc pas un simple retour au cadre national ; ils suggèrent de saisir les trajectoires de construction et d'affirmation des États-nations à partir de leurs dynamiques transnationales. L'ouverture de l'Allemagne de l'Est, que ce soit par la pratique des jumelages au sein du bloc de l'Est ou par la valorisation de l'expérience internationaliste des militants communistes, participe, non sans tensions, au processus symbolique de construction nationale dans l'après-guerre.

Si elle permet de déconstruire la rhétorique communiste internationaliste, l'approche transnationale laisse cependant ouverte la question des conditions d'émergence de véritables « cultures internationales », façonnées ou redéfinies par l'expérience communiste ${ }^{20}$. C'est tout l'intérêt de l'article de Simon Godard qui ouvre une réflexion sur ce sujet, en partant non pas des discours mais des acteurs. Centrée sur les fonctionnaires est-allemands du Conseil d'aide économique mutuelle (CAEM), l'organisation chargée de coordonner les économies nationales au sein du bloc de l'Est, l'enquête biographique qu'il propose examine

19. Voir, par exemple, Saskia Sassen, La globalisation. Une sociologie, Paris, Gallimard, 2009 (2007).

20. Sur cette notion de « culture internationale», entendue à la fois comme un mode de vie et un ensemble spécifique de compétences et de connaissances, voir Anne-Catherine Wagner, Les nouvelles élites de la mondialisation. Une immigration dorée en France, Paris, PUF, 1998. 
les différents usages que les acteurs font des ressources internationales dans le monde socialiste et définit une typologie des experts de l'économie socialiste en fonction des rapports qu'ils entretiennent avec l'échelle internationale. Il montre ainsi comment, au cours des années 1960, les fonctionnaires du CAEM commencèrent à former un groupe social uni par des liens de sociabilité intenses à Moscou et partageant des compétences professionnelles spécifiques. Certes, l'État était toujours opératoire dans l'horizon d'attente de ces fonctionnaires internationaux, mais il l'était sur un mode non exclusif : pour certains d'entre eux, le bloc de l'Est devint un référentiel pertinent qui permettait l'émergence d'une « économie-monde socialiste ».

\section{Repenser les chronologies de la " globalisation »}

Autre implicite des approches transnationales que questionnent les auteurs de ce dossier, celui de l'exceptionnalité de l'épisode communiste. Dans les récits de la mondialisation, le « court $\mathrm{XX}^{\mathrm{e}}$ siècle $»^{21}$ est bien souvent présenté comme une parenthèse, comme cadré, en amont, par la vague d'internationalisation de l'économie de la fin du XIX ${ }^{\mathrm{e}}$ siècle et, en aval, par la fin de l'Union Soviétique. Dans son livre intitulé The World Is Flat, qui connut un certain succès au début des années 2000, le journaliste américain Thomas Friedman défend par exemple la thèse d'un « aplatissement » du monde dû à une série de facteurs, parmi lesquels figure, en premier lieu, l'effondrement du bloc de l'Est. La chute du Mur de Berlin aurait ainsi mécaniquement levé les barrières ${ }^{22}$. Les obstacles aux relations internationales, dans le domaine économique notamment, seraient tombés, favorisant une nouvelle étape du processus de mondialisation et l'émergence d'une pensée globale. Cette rupture est devenue un leitmotiv des discours sur la mondialisation, sans que l'articulation supposée entre frontière concrète, matérielle, et frontières mentales soit interrogée. Or poser cette question peut conduire à repenser les temporalités du XXe siècle ou la rupture de 1989-1991, comme le suggèrent ici Larissa Zakharova et Ioana Cîrstocea.

De fait, en examinant la place de l'Union Soviétique dans les réseaux de télécommunication internationaux, L. Zakharova propose une nouvelle chronologie non pas ponctuée par les événements politiques, mais informée par le type d'interaction entre l'Est et l'Ouest. Alors que durant l'entre-deux-guerres, et même lors de la phase de stalinisation de l'Union Soviétique, des formes ouvertes de coopération étaient possibles et permettaient une « circularité » dans les itinéraires Est-Ouest des techniques, la seconde guerre mondiale, avec la récupération massive de biens et de techniques allemands, inaugura un nouveau « paradigme prédateur ». Dès

21. Selon le titre de l'ouvrage de Eric Hobsbawm, L'âge des extrêmes. Histoire du court XXe siècle, 1914-1991, Bruxelles, Éditions Complexe/Le Monde diplomatique, 1999 (1994).

22. Thomas L. Friedman, La terre est plate. Une brève histoire du XXI siècle, Paris, Perrin, 2010, p. 49-55 notamment (The World Is Flat: A Brief History of the Twenty-First Century, New York, Farrar, Straus and Giroux, 2005). 
lors, les techniques suivirent une trajectoire linéaire et unidirectionnelle (de l'Ouest vers l'Est) et ne correspondirent plus qu'à de simples transferts. Cette distinction conceptuelle (circulation/transfert) permet de réévaluer la rupture introduite par la déstalinisation et le Dégel khrouchtchévien : malgré une politique diplomatique tournée vers «la coexistence pacifique », le discours soviétique sur la prétendue supériorité du socialisme et les frontières mentales qu'il dessine entre l'Est et l'Ouest interdisent bien souvent de véritables circulations techniques semblables à celles des années 1930.

C'est à une révision comparable de la chronologie qu'aboutit I. Cîrstocea, à partir de son enquête sur la formation, dans les années 1990, d'un réseau féministe transatlantique, le Network of East-West Women. Ce mouvement fut en effet impulsé par des féministes américaines politisées à gauche et désireuses de profiter des opportunités créées par l'effondrement de l'Union Soviétique pour renouveler leur militantisme. Malgré cette volonté d'ouverture, le NEWW contribua à réactiver la division Est-Ouest, en faisant de la catégorie des « femmes est-européennes » un support symbolique de leur action politique. I. Cîrstocea montre ainsi comment les cadrages idéologiques de la guerre froide continuèrent, bien après la fin du communisme, à façonner les interactions Est-Ouest. Ils furent réactivés par tous ces « entrepreneurs de démocratie », d'impulsion gouvernementale (américaine notamment) ou non (les fondations philanthropiques, par exemple), qui, à partir des années 1990, investirent politiquement l'opposition Est-Ouest pour promouvoir et exporter les droits et normes du libéralisme dans les pays anciennement socialistes. Ainsi, les frontières mentales Est-Ouest pourraient finalement paraître plus prégnantes dans les années 1990 qu'elles ne l'étaient trente ans plus tôt, à un moment où, certes, les tensions diplomatiques et militaires entre les deux blocs demeuraient fortes, mais où la thèse de la « convergence des systèmes », portée à ses débuts par les sociologues américains (Talcott Parsons, Daniel Bell, notamment ${ }^{23}$ ), fut largement diffusée ${ }^{24}$. Le discours sur « la société post-industrielle », vers laquelle les sociétés soviétique et occidentale étaient censées évoluer, contestait le poids de l'idéologie. Cette dépolitisation permettait de définir des signes de reconnaissance autour de problèmes communs ou d'une vision partagée de l'économie ${ }^{25}$, de la modernité et du futur ${ }^{26}$, accompagnant ainsi l'ouverture internationale des élites (intellectuelles, économiques et politiques) du bloc de l'Est. Avec cette redéfinition

23. D. C. Engerman, Know Your Enemy. The Rise and Fall of America's Soviet Experts, New York, Oxford University Press, 2009, chap. 7.

24. Sur le rôle des fondations philanthropiques américaines dans la diffusion de ce discours, voir Nicolas Guilhot, « "Un réseau d'amitiés agissantes" : les deux vies de la Fondation pour une entraide intellectuelle européenne (1957-1991) », dans Ludovic Tournès (dir.), L'Argent de l'influence. Les fondations américaines et leurs réseaux européens, Paris, Autrement, 2010, p. 164-184.

25. Johanna Bockman, Markets in the Name of Socialism. The Left-Wing Origins of Neoliberalism, Stanford, Stanford University Press, 2011.

26. C'est sur cette hypothèse que le projet Futurepol coordonné par Jenny Andersson, à Sciences Po, travaille, à partir d'une enquête transnationale sur l'émergence et le développement d'un champ d'expertise sur la prévision et la futurologie. Jenny Andersson, Églé Rindzevičiūté, « The Political Life of Prediction. The Future as a Space of Scientific World Governance in the Cold War Era », Les Cabiers européens de Sciences Po, 4, décembre 2012. 
de la carte mentale du monde, une nouvelle philosophie du social s'est diffusée. En gommant les clivages politico-idéologiques Est-Ouest, elle a permis d'imposer, dès les années 1960-1970, une vision unifiée et pacifiée de la société et de conforter un nouveau registre de légitimité fondé sur la science, l'expertise, l'économie ${ }^{27}$.

$\grave{A}$

rebours de l'image optimiste d'un monde connecté et uni ou d'un discours positif sur la mondialisation, largement véhiculés par les approches globales ${ }^{28}$, ce dossier souligne combien les pouvoirs délimitent et définissent les frontières aussi par leurs pratiques discursives. Il invite à replacer au cœur de l'analyse la question de la domination et de l'État, de ses registres de légitimité ou de ses modes de gouvernement, et propose ainsi une réflexion sur la façon dont l'ordre politique se maintient et se redéfinit. C'est bien, en effet, avec cette question que renoue la perspective transnationale, dès lors qu'elle est mobilisée pour étudier ces contextes « résistants aux contacts externes » que furent les configurations de circulations façonnées par l'expérience communiste ${ }^{29}$.

Paul Boulland est chercheur associé (CNRS) au Centre d'histoire sociale du XX $X^{\mathrm{e}}$ siècle et co-directeur du Dictionnaire biographique, mouvement ouvrier, mouvement social (19401968) (Le Maitron). Il est l'auteur d'une thèse de doctorat sur l'encadrement du PCF de la Libération aux années 1970 (2011, Université Paris 1), et a notamment publié "Sortir du rang ? Rapports à l'usine des cadres ouvriers communistes ", Actes de la recherche en sciences sociales (196-197, 2013, p. 54-71). Ses recherches actuelles portent sur la professionnalisation du syndicalisme en Europe et en France.

paul.boulland@orange.fr

Isabelle Gouarné est chargée de recherche au CURAPP-ESS (CNRS-Université Jules Verne de Picardie). Ses recherches portent sur I'histoire des sciences sociales et la circulation transnationale des idées. Elle a publié récemment L'introduction du marxisme en France. Philosoviétisme et sciences humaines, 1920-1939 (Rennes, Presses universitaires de Rennes, 2013) et «Engagement philosoviétique et posture sociologique dans l'entre-deux-guerres: le rôle politico-intellectuel de Georges Friedmann " (Sociologie du travail, 54 (3), 2012, p. 356-374).

isabelle_gouarne@hotmail.com

27. Pour la France, voir Delphine Dulong, Moderniser la politique : aux origines de la Ve République, Paris, L'Harmattan, 1997.

28. H.-G. Haupt, « Une nouvelle sensibilité : la perspective "transnationale”. Une note critique », art. cité. 29. Ce dossier est issu d'une journée d'études organisée en juin 2012 grâce au soutien du Centre d'histoire sociale du XX $\mathrm{XX}^{\mathrm{e}}$ siècle et de l'équipe CSU du CRESPPA. Nos vifs remerciements vont aux participants de cette journée, notamment aux membres de son comité scientifique (Sophie Cœuré, Yves Cohen, Susan Gross Solomon, Claude Pennetier et Bernard Pudal), aux discutants (José Gotovitch et Liora Israël), ainsi qu'aux relecteurs de Critique internationale, qui tous ont grandement contribué à l'enrichissement de notre réflexion. 